\title{
Register rerum ad vol. 113
}

Demonstrationen * Demonstrations * Demonstrations *

Buchbesprechungen *** Book Reviews *** Livres Nouveaux ***

Originalarbeiten ohne Bezeichnung Original articles without asterisk Travaux originaux sans designation

Sammelreferate **

Surveys **

Revues Générales **

Acanthose, influence du mode de preparation d'un onguent sur la 35

ACTH-Intoleranz 306*

Adenolymphom 304*

Akne vulgaris $61 * *$

Akrodermatitis chronica atrophicans $60 * *$

Akrodermatitis continua Hallopeau $53 * *$

Aktinomykosen $183^{* *}$

Allergie, Therapie akuter lebensbedro-hender Formen der 300

Alternde Haut $63 * * *$

Anetodermia maculosa $60 * *$

Aneurysm, cirsoid, in the skin 129

Antibiotica et Chemotherapia $406 * * *$

Antibiotiques, Accidents à levures dans les traitements par les 189***

Arachnida, Hautveränderungen durch $331 * *$

Arsthinol (Balarsen) in Dermatology 369

Arzneimittel, dermatologische $333 * * *$

Berufliche Hautkrankheiten, prophy-laktische Maßnahmen zur Verhütung der 255

Blastomykose, brasilianische $184 * *$

Blastomykose, nordamerikanische $184 * *$

Blastomykosen $183 * *$

Brucellosen $185^{* *}$

Calcinosis interstitialis $309 *$ Cheilitis granulomatosa $94 * *$ Chemotherapia et Antibiotica 406*** Chirurgie der Haut $64 * * *$ Chromoblastomykosis $184 * *$

Civatte, Dr. A.; Nekrologia 190

Colibacülosis cutanea $185^{* *}$

Complesso ungueale, Fisio-Patologia del $63 * * *$

Condyloma accuminatum $188^{* *}$

Contact Dermatitis from Neomycin due to Dermal delayed (Tuberculin-type) Sensitivity 191

Cycloheximid-(Actidion)-Agar, Züchtung von Dermatophyten auf 65

Dermatitis herpetiformis 52**

Dermatitis herpetiformis 309* 


\section{Dermatomykosen $179 * *$}

Dermatomykosen, Studien zur Epide-miologie und Therapie der 156

Dermatomyositis $59 * *$

Dermatophyten, Züchtung auf Cyclo-heximid- (Actidion-) Agar, ein Fort-schritt 65

Dermatosen, bedingt durch ektogene und endogene akzidentelle Einflüsse 107**

Diabetes mellitus und Porphyria cutanea tarda, Ätiologie 78

Eczema experimental 335 Ekzematogenic Agent in Turpentine Oil, on the Chemical Nature of II 1 Elastoma intrapapillare perforans ver-

ruciforme (Miescher) 13 Erysipel 185** Erythema axillarum perstans of micro-

coccal origin 202 Erythematodes $55^{* *}$

Register rerum

409

Framboesia 249**

Fußmykosen, Studien zur Epidemiolo-gie und Therapie der 156

Genitalien, nichtvenerische Krankheiten

der64*** Geschleclitskrankheiten, Lehrbuch der

$407 * * *$ Granuloma anulare $97 * *$ Granulomatosis disciformis chronica et

progressiva $101 * *$ Granulomatosis disciformis chronica et

progressiva $315^{*}$ Granulome, teleangiektatische $312 *$ Griffes de chat, Maladie de $187 * *$

Hautchirurgie 64*** Haut- u. Geschlechtskrankheiten, Lehrbuch der 332*** Haut- und

Geschlechtskranhkeiten,

Lehrbuch der 406*** Hautkrankheiten, Heutiger Stand der

Therapie der 334*** Hautkrebse , Hyaluronsäure - Untersu-

suchungen an lokalen Myxödemen

und im Stroma der 219 Hautschutz, gewerblicher, bei Coiffeu-

ren 278 Hautschutz, Die Grenzen des 292 Hautschutz mittels sogenannter Haut-

schutzcremen 284 Hautschutzsalben,Untersuchungenüber

die Nützlichkeit der 260 Hautveränderungen, bedingt durch ek-

togene und endogene akzidentelle

Einflusse $107^{* *}$ Hautveränderungen, Durch wirbellose

Tiere erzeugte $331 * *$ Heihnittel 1950-1955, Die äußeren

$333^{* * *}$ Herpes simplex recidivans 186** Herpes zoster 186** Hexapoda, Hautveränderungen durch

331** Histoplasmosis 184** Hyaluronsäure-Untersuchungen an lokalen Myxödemen und im

Stroma

der Hautkrebse 219

Hyperkeratosis Palmo-Plantaris with Peridontosis (Papillon-Lefèvre) 207

Immunologie générale, Elements de Impetigo herpetiformis $53 * *$

Katzenkrankheit $187 * *$

Keratosis follicularis epidemica $62 * *$

Keratosis follicularis serpiginosa (Lutz)

13 Kontaktekzem, experimentelles 335 Kontaktekzem durch Neomycin, bedingt durch dermale (delayed Tuberculin-type) Überempfindlichkeit 191

Largactilekzeme 307* LE-Zell-Phänomen, Zur Frage der Spe-

zifität des 375 Leishmaniasis, cutaneous (Oriental sore 
243** Leishmaniasis, dermal, Post-Kala-Azar

$246^{* *}$ Leishmaniasis, Muco-Cutaneous (American) 243** Leprosy, trophic ulcers of the feet in, Treatment with Ergot alcaloids 142 Leukemia, lymphatic and scabies nor-

vegica 26 Levures, accidents à, dans les traitements par les antibiotiques $189 * * *$ Lichen nitidus $97 * *$ Lichen ruber planus $47 * *$ Lichen sclerosus et atrophicus $59 * *$ Lupus erythematodes $55^{* *}$ Lupus erythematodes $307 *$ Lupus erythematodes der Conjunctiva,

Chronischer 226 Lymphogranuloma inguinale 187** Lymphozytom 304*

Maduromykosen $183 * *$

Makrocheilie, essentielle granulomatöse

94** Melanom 315* Melkerpocken 187** Melkersson-RosenthaГsches Syndrom $94 * *$

Molluscum contagiosum 188**

410

Register rerum

Morbus Behçet 188*

Morbus Besnier-Boeck-Schaumann 88**

Morbus Darier 315*

Morbus Fiessinger-Leroy-Reiter 188**

Mykosen179**

Mykosen, Studien zur Epidemiologie und

Therapie der Fußmykosen 156 Myxödem, lokales, Hyaluronsäure-Un-

tersuchungen im Stroma der Haut-

krebse und an 219

Nagel, Physiopathologie des $63 * * *$ Necrobiosis lipoidica $99 * *$ Neurodermitis $43 * *$

Ottenstein, Frau Prof. Berta; Nekrologia 190

Panniculitis $101 * *$

Parapsoriasis $58^{* *}$

Pemphigus und Pemphigoide $49 * *$

Phlebitiden, oberflächliche, strangför-

mige $315^{*}$ Pinta251**

Plattenepithelkarzinom 313* Pigment 103** Pityriasis rubra pilaris 58** Poikilodermia

atrophicans vascularis

$60 * *$ Porphyria cutanea tarda und Diabetes

mellitus, Atiologie 78

Prurigo $46^{* *}$

Pruritus $40^{* *}$

Psoriasis $53 * *$

Psoriasis and Stress 71

Purpura $118^{* *}$

Pustulosis varicelliformis Kaposi $186^{* *}$

Pyodermien $184 * *$

Radiothérapie en Dermatologie $62 * * *$ Rhinosklerom 185** Rosacea $62 * *$

Sag $1 / 8$ er, Prof. Felix; Personalia 190 Scabies Norvegica and Lymphatic Leukemia 26 Schuppli, Prof. Dr. Rudolf; Personalia 190 
Schweizerische Gesellschaft für Dermatologie und Venereologie. Colloquium Dermatologicum. Aarau, 29. April 1956: Mitteilungen 300-303, Demon-strationen 304*-316*, Diskussion 294-299 Sebum, Role protecteur du 288

Senescenza della cute umana $63 * * *$

Sézary, Dr. A., Necrologia 407

Skin surgery $64 * * *$

Sklerodermie $58 * *$

Société Suisse de Dermatologie et de Vénéréologie. Colloquium Dermatologicum. Aarau, 29

avril 1956: Communications 300-303, Demonstrations 304*-316*, Discussion 294-299

Sporotrichose $183^{* *}$

Stachelzellkrebs der Lippen $311^{*}$

Strahlenheilkunde 390**

Syphilis $316^{* *}$

Syphilis, endemic $252 * *$

Talg, Hautschutz durch 288 Terpentinekzem 1 Treponematoses 246** Tuberkulose der Haut 232** Tumoren der Haut. Hyaluronsäure-

Untersuchungen an lokalen Myxöde-

men und im Stroma der Hautkrebse

219 Turpentine Oil, On the Chemical Nature

of the Eczematogenic Agent in, II 1

Ulcer, tropical $253 * *$

Ulcers, trophic, of the soles of the feet in leprosy. Treatment with Ergot Alkaloids 142

Ultra-violets, Le rayons en Médecine 407***

Vakzine $186^{* *}$

Verrucae vulgares $187 * *$ Virus-Erkrankungen $186 * *$

White spot disease $59 * *$

Würmer, Hautveränderungen durch

$332 * *$

Yaws (Framboesia) 249** 